Review

\title{
Extended upper abdominal resections as part of debulking surgery at the time of tertiary cytoreduction for relapsed ovarian cancer; case report and literature review
}

\author{
Nicolae Bacalbașa ${ }^{1}$, Irina Balescu ${ }^{2}$ \\ ${ }^{1}$ Carol Davila University, Department of General Surgery, Bucharest, Romania \\ ${ }^{2}$ Ponderas Clinical Hospital, Department of General Surgery, Bucharest, Romania
}

\begin{abstract}
Ovarian cancer remains one of the most aggressive gynecologic malignancies with high capacity to recur even in cases submitted to surgery with curative intent. However, even in these cases the best therapeutic option in order to achieve a good control of the disease remains radical surgery.

We present the case of a 65-year-old patient diagnosed submitted to surgery for stage IIIC ovarian cancer five years before. At two years follow up she was diagnosed with an isolated recurrence at the level of the hepatic pedicle which was successfully removed. At 18 months follow up she was diagnosed with a large recurrence in the left superior abdominal quadrant and a liver metastasis which were resected. At 18 months follow up she is free of recurrent disease.
\end{abstract}

Keywords: advanced stage ovarian cancer, tertiary cytoreduction, debulking surgery 


\section{Introduction}

Ovarian cancer is an aggressive gynecological malignancy with a significant capacity of relapse even if complete cytoreduction to no residual disease is performed $(1,2)$. This aspect is related to an aggressive biological behavior and with the capacity of the tumor cells of spread using multiple patterns, including peritoneal, lymphatic and haematogenous route. However, even in cases in which recurrence is diagnosed debulking surgery remains the therapeutic golden standard. When it comes to the upper abdominal involvement, although initially it has been considered that cases presenting upper abdominal tumor burden cannot be candidates for debulking surgery, improvement of the surgical techniques and of the postoperative management leaded to a widely introduction of these procedures as part of debulking surgery $(3,4)$.

\section{Case report}

The 65-year old patient had been initially investigated for pelvic pain and weight loss and was diagnosed at that moment with stage IIIC ovarian cancer. The patient was submitted to debulking surgery, a total hysterectomy with bilateral adnexectomy, pelvic, para-aortic lymph node dissection, pelvic and parietal peritonectomy and omentectomy being performed (Figures 1-3).

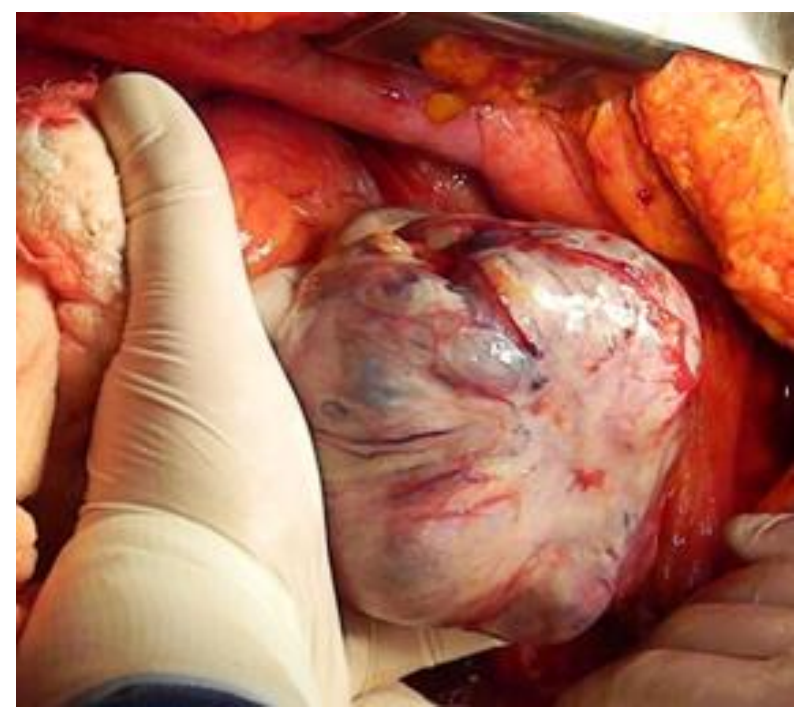

Figure 1. Large ovarian tumor - initial aspect at the moment of primary cytoreduction

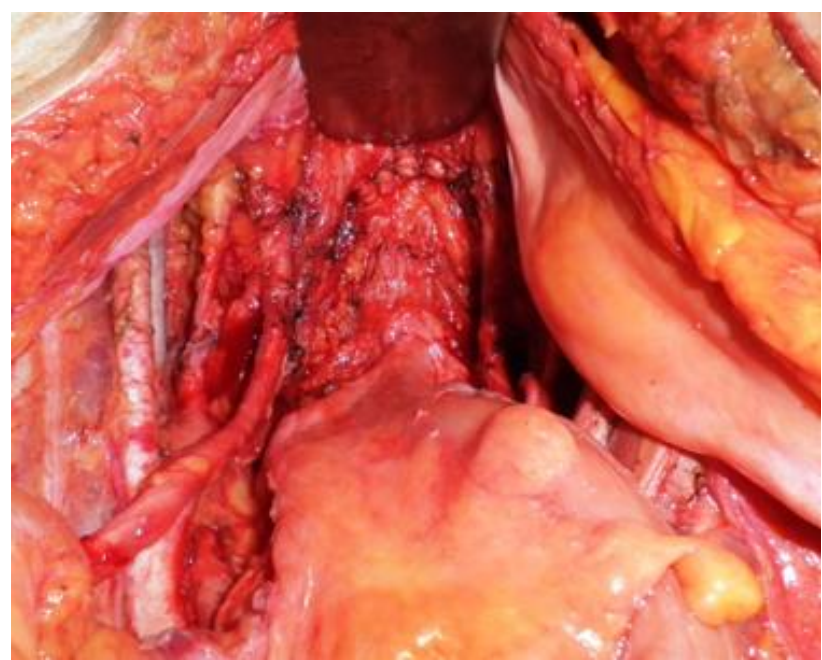

Figure 2. The final aspect after total hysterectomy with bilateral adnexectomy, pelvic and parietal epritonectomy, pelvic and para-aortic lymph node dissection

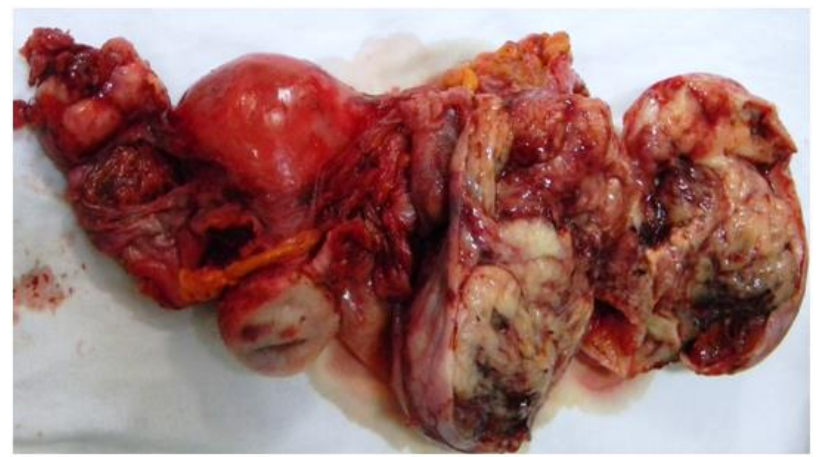

Figure 3. The specimen of total hysterectomy with bilateral adnexectomy

At the time of primary cytoreduction debulking to no residual disease was achieved. The histopathological studies revealed the presence of a poorly differentiated serous ovarian carcinoma. One month postoperatively the patient was submitted to six cycles of adjuvant chemotherapy with platinum slats and taxanes regimens. At two years follow up the patient was diagnosed with an isolated adenopathic mass located at the level of the hepatic pedicle. At that moment she was submitted to secondary cytoreduction consisting of lymph node dissection at the level of the hepatic pedicle (Figure 4). The histopathological studies confirmed again the presence of metastatic serous ovarian carcinoma cells. Three weeks after discharged the patient was readdressed to the oncology clinic where she was submitted to six cycles of 
adjuvant chemotherapy. At 18 months follow up the patient was diagnosed with two recurrent tumors: an isolated liver metastasis developed in segment $\mathrm{V}$ according to Couinaud's classification and a concurrent lesion developed in the left superior abdominal quadrant involving the great gastric curvature, the spleen and the tail of the pancreas (Figures 5-8).

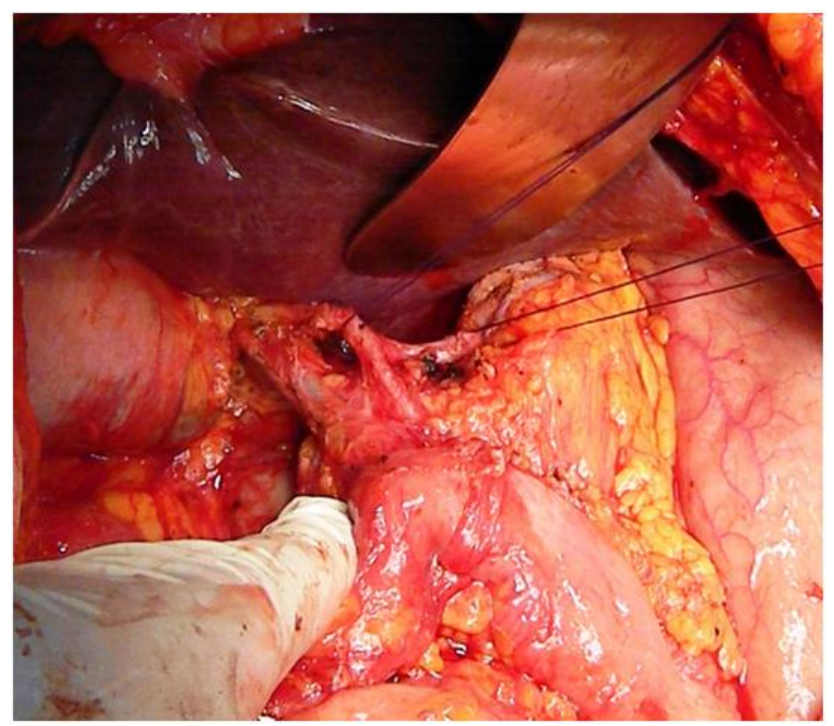

Figure 4. Lymph node dissection in the hepatic pedicle at the time of secondary cytoreduction

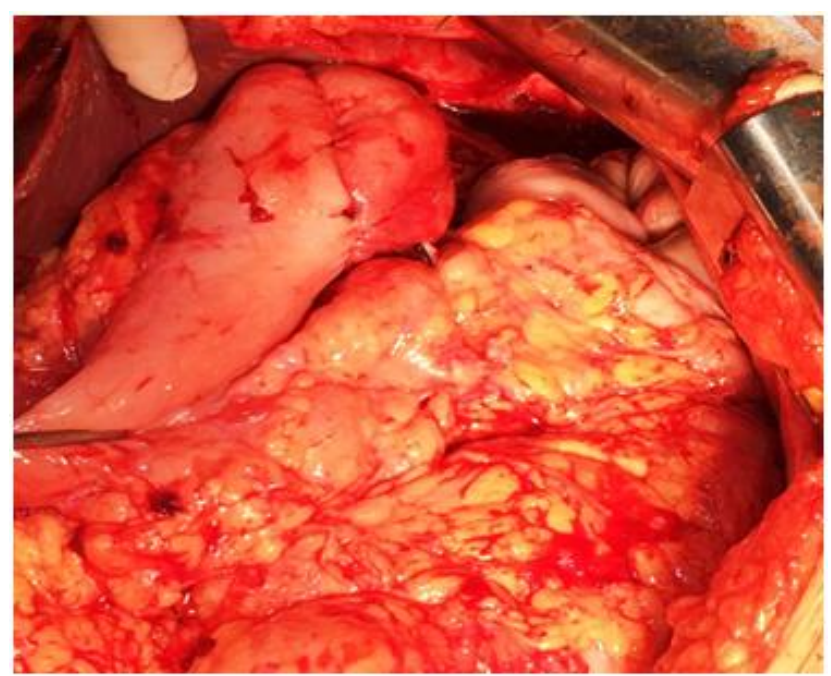

Figure 5. large upper abdominal recurrence invading the pancreatic tail the stomach and the spleen at the moment of tertiary cytoreduction

The lesion was resected en bloc with the invaded areas. The patient developed a grade $\mathrm{C}$ pancreatic fistula which necessitated reoperation; the post reinterventional course was uneventful; the patient being discharged in the 14th postoperative day. The histological studies confirmed the metastatic origin of the tumor. Two months after discharged the patient was re-submitted to adjuvant chemotherapy. At 18 months follow up after tertiary cytoreduction she is alive and free of recurrence.

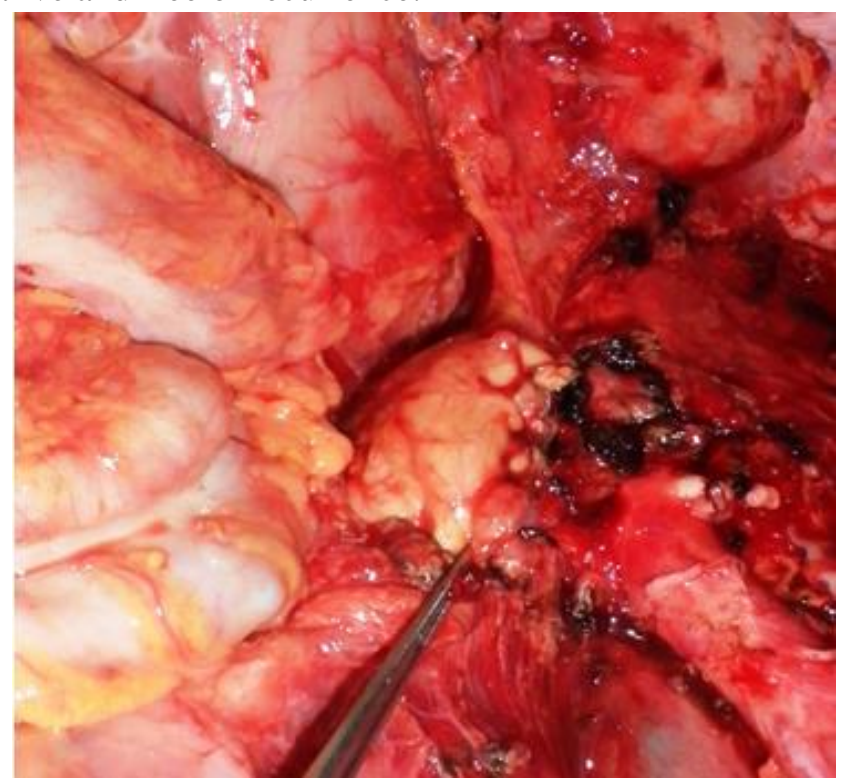

Figure 6. The final aspect after resection: the recurrence was resected en bloc with distal pancreatectomy, parcelar gastrectomy and splenectomy

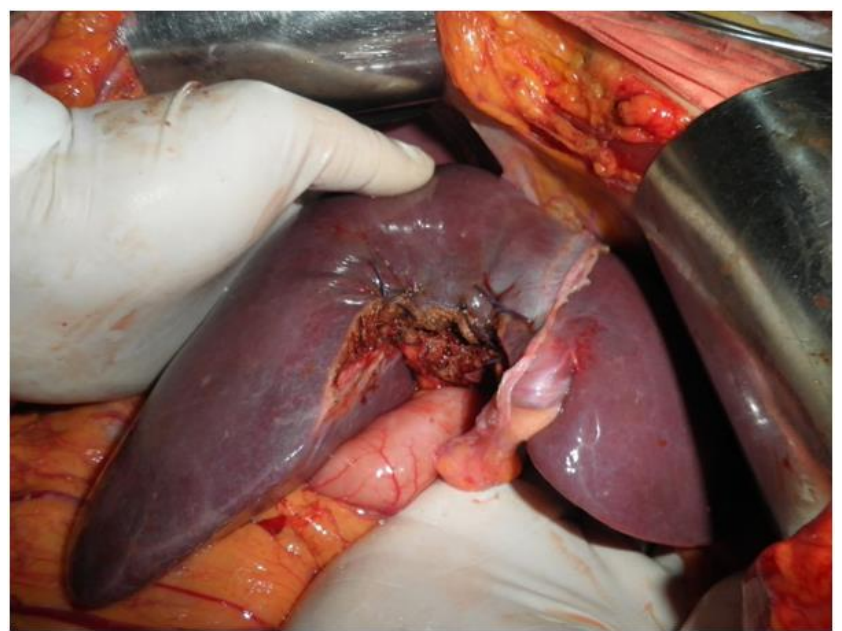

Figure 7. Atypical hepatectomy for isolated liver metastasis - tertiary cytoreduction

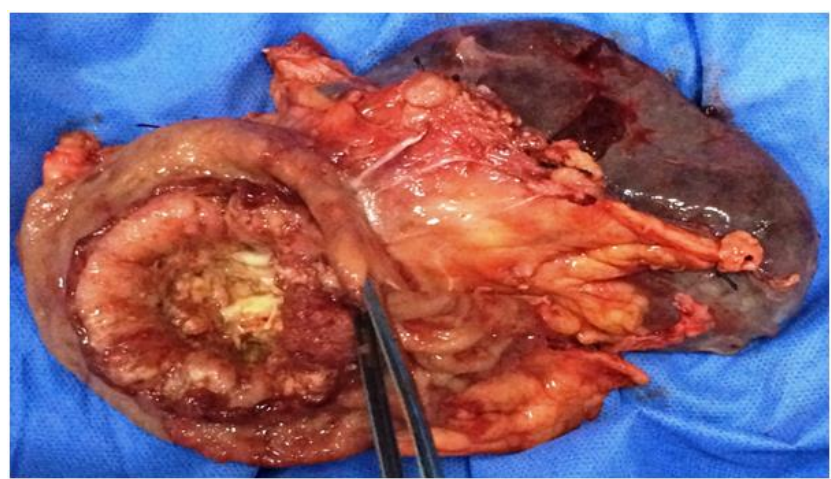

Figure 8. The specimen of parcelar gastrectomy, distal pancreatectomy and splenectomy (removed at the moment of tertiary cytoreduction) 


\section{Discussions}

Ovarian cancer is the fifth cause of cancer related death among women worldwide, this data being justified by the fact that most women are diagnosed in an advanced stage of the disease. However, the prognosis of patients with advanced stage disease significantly improved after demonstrating the benefits of debulking surgery followed by adjuvant platinum based therapy. Although after performing this therapeutic sequence up to $90 \%$ of patients will report a complete clinical response, relapse is to be expected in $70-80 \%$ of cases. When it comes to the best therapeutic strategy in recurrent ovarian cancer studies have shown that debulking surgery is de nouveau the most efficient procedure in order to achieve a good control of the disease (5, 6). However, even after secondary cytoreduction recurrence might develop, so the concept of tertiary cytoreduction has been proposed.

One of the first papers which reported the benefits of tertiary cytoreduction was published in 2004, being conducted by Leitao et al; the authors demonstrated that the long term outcomes after tertiary cytoreduction are mostly influenced by a longer than 12 months' time to first recurrence $(\mathrm{p}=0,04)$, by a treatment free interval longer than 12 months $(\mathrm{p}=0,002)$ and by an optimal debulking surgery at the moment of tertiary cytoreduction. Leitao's study was conducted on 26 patients submitted to tertiary cytoreduction between 1/1/90 and 12/31/2002 at Memorial Sloan Kettering Cancer Center and involved multiple visceral resections such as bowel resections (in eight cases), hepatic resections (in three cases), diaphragmatic resections (in two cases), urinary tract resections (nephrectomy in one case and partial cystectomy in one case) and pelvic or paraaortic lymph node dissection. In the meantime, regarding the rates of postoperative morbidity, seven patients developed complications. However, there were only two grade 3 complications (intraabdominal abscesses necessitating percutaneous drainage) and no complication of grade 4 or 5 . The authors concluded that tertiary cytoreduction is associated with favorable outcomes and acceptable rates of postoperative complications (7).

In order to continue the study of Leitao on a larger subgroup of patients, Shih et al conducted a study on 77 patients submitted to tertiary cytoreduction at Gynecology Service, Department of Surgery, Memorial Sloan-Kettering Cancer Center, USA between 1998-2008. Tertiary cytoreduction was performed after a median interval of 25,7 months from the moment of secondary cytoreduction while the median number of chemotherapy cures prior to tertiary cytoreduction was two (range 1-5); when it comes to the radicality of secondary cytoreduction, it was estimated that optimal cytoreduction (defined as residual tumor $<0,5 \mathrm{~cm}$ ) had been achieved in $92,2 \%$ of cases. The authors studied the prognostic factors in order to predict an improved survival and demonstrated that the overall survival was increased in patients presenting isolated recurrences (single versus multiple $-\mathrm{p}=0,02$ ), time to secondary cytoreduction $>24$ months $(\mathrm{p}<0,01)$, treatment free interval $>12$ months $(\mathrm{p}=0,02)$, platinum sensitivity $(\mathrm{p}=0,005)$ and lower values of CA 125 at the time of tertiary cytoreduction $(p=0,004)$. In this way the authors demonstrated the benefits of performing aggressive surgical procedures as part of tertiary cytoreduction in order to improve the outcomes of these patients and stated that it should be performed especially in cases in which cytoreduction to no gross residual disease is feasible (8). 
A similar study was conducted by Hizli et al and published in 2011. In this study the authors introduced 23 patients submitted to tertiary cytoreduction between January 1999 and January 2011. The most important factor associated with an improved outcome after tertiary cytoreduction was the absence of residual disease at the end of tertiary cytoreduction. However, the other parameters which were positively correlated with an improved survival in Shih's study (the preoperative values of $\mathrm{CA} 125$, treatment free interval) did not have statistical significance; in the meantime, the criterion of platinum sensitivity was omitted from the beginning, one of the inclusion criteria in Hizli's study being the presence of platinum sensitive tumors $(9,10)$.

\section{Conclusions}

Tertiary cytoreduction is perfectly justified in order to achieve an increase in terms of survival for patients with recurrent ovarian cancer especially in cases in which cytoreduction to no gross residual disease is estimated. In the meantime, the presence of disseminated lesions necessitating multiple visceral resections should not constitute an obstacle for achieving an $\mathrm{R}_{0}$ resection. However, larger studies are still necessary in order to establish which the perfect candidates for tertiary cytoreduction are.

\section{References}

1. Jemal A, Siegel R, Ward E, Hao Y, Xu J, Murray T, Thun MJ. Cancer statistics, 2008. CA Cancer J Clin. 2008; 58(2): 71-96. PMID: 18287387, https://doi.org/10.3322/CA.2007.0010

2. Eisenkop SM, Friedman RL, Wang HJ. Complete cytoreductive surgery is feasible and maximizes survival in patients with advanced epithelial ovarian cancer: a prospective study. Gynecol Oncol. 1998; 69(2): 103-8. PMID: 9600815, https://doi.org/10.1006/gyno.1998.4955
3. Khatib G, Guzel AB2, Gulec UK2, Vardar MA2. A novel technique: Carbon dioxide gas-assisted total peritonectomy, diaphragm and intestinal meso stripping in open surgery for advanced ovarian cancer (Çukurova technique). Gynecol Oncol. 2017; 146(3): 674-5. PMID: 28720378, https://doi.org/10.1016/j.ygyno.2017.07.132

4. Chi DS, Eisenhauer EL, Lang J, Huh J, Haddad L, Abu-Rustum NR, Sonoda Y, Levine DA, Hensley M, Barakat RR. What is the optimal goal of primary cytoreductive surgery for bulky stage IIIC epithelial ovarian carcinoma (EOC)? Gynecol Oncol. 2006; 103(2): 559-64. PMID: 16714056, https://doi.org/10.1016/j.ygyno.2006.03.051

5. Rose PG, Java JJ, Morgan MA, Alvarez-Secord A, Kesterson JP, Stehman FB, Warshal DP, Creasman WT, Hanjani P, Morris RT, Copeland LJ. Disease extent at secondary cytoreductive surgery is predictive of progression-free and overall survival in advanced stage ovarian cancer: An NRG Oncology/Gynecologic Oncology Group study. Gynecol Oncol. 2016; 143(3): 511-5. PMID: 27692669,

https://doi.org/10.1016/j.ygyno.2016.09.005

6. Dessapt AL, Huchon C, Ngo C, Bats AS, Bensaid C, Lecuru F. Is complete cytoreductive surgery feasible in this patient with ovarian cancer? Surg Oncol. 2016; 25(3): 326-31. PMID: 27566040, https://doi.org/10.1016/j.suronc.2016.07.001

7. Karadayi K, Yildiz C, Karakus S, Akkar OB, Ugurlu GP, Kurt A, Yanik A. Cytoreductive surgery and perioperative intraperitoneal chemotherapy for gynecological malignancies: a single center experience. Eur J Gynaecol Oncol. 2016; 37(2): 194-8. PMID: 27172744

8. Shih KK, Chi DS, Barakat RR, Leitao, MM. Tertiary cytoreduction in patients with recurrent epithelial ovarian, fallopian tube or primary peritoneal cancer. Gynecol Oncol. 2010; 117(2): $\quad 330-5 . \quad$ PMID: 20189234 , https://doi.org/10.1016/j.ygyno.2010.01.046 
9. Hızlı D, Boran N, Yılmaz S, Turan T, Altınbaş SK, Celik B, Köse MF. Best predictors of survival outcome after tertiary cytoreduction in patients with recurrent platinum-sensitive epithelial ovarian cancer. Eur J Obstet Gynecol Reprod Biol. 2012; 163(1): 71-5. PMID: 22480413, https://doi.org/10.1016/j.ejogrb.2012.03.018
10. Pölcher M, Zivanovic O, Chi DS. Cytoreductive surgery for advanced ovarian cancer. Womens Health (Lond). 2014; 10(2): 179-90. https://doi.org/10.2217/WHE.14.4 